\title{
Les résultats exceptionnels de lasi, sondeur atmosphérique hyperspectral de Metop
}

\section{Résumé}

La deuxième conférence internationale Iasi (Infrared Atmospheric Sounding Interferometer), qui s'est tenue à Sévrier (Haute-Savoie) du 24 au 29 janvier 2010, a rassemblé 150 scientifiques de tous horizons pour faire le point sur l'instrument Iasi, le traitement des données, les résultats de son exploitation, trois ans après sa mise en service, et pour envisager les suites du programme. Dans cet article, on présente l'instrument monté sur les trois satellites Metop d'Eumetsat qui se succéderont jusqu'en 2020. On illustre succinctement les extraordinaires performances de cet instrument devenu référence pour l'interétalonnage de capteurs infrarouges. Après un rappel sur l'organisation du traitement des données, les perspectives à court et moyen termes sont mises en lumière. La partie principale traite des résultats les plus récents exposés à la conférence dans les différents domaines d'application de Iasi : la prévision numérique, l'étude de la composition atmosphérique, les applications opérationnelles de la chimie atmosphérique et le suivi du changement climatique. Le dernier paragraphe est consacré au futur avec les évolutions attendues des produits et les perspectives de l'après Iasi avec le sondeur infrarouge sur Météosat troisième génération et le sondeur Iasi nouvelle génération.

\author{
T. Phulpin ${ }^{(1)}$, C. Camy-Peyret ${ }^{(3)}$, J. Taylor ${ }^{(2)}$, C. Clerbaux ${ }^{(4)}$, P. Coheur ${ }^{(5)}$, \\ C. Crevoisier ${ }^{(6)}$, D. Edwards ${ }^{(7)}$, A. Gambacorta ${ }^{(8)}$, V. Guidard ${ }^{(9)}$, \\ F. Hilton ${ }^{(2)}$, N. Jacquinet ${ }^{(6)}$, R. Knuteson ${ }^{(10)}$, L. Lavanant $^{(9)}$, T. McNally ${ }^{(10)}$, \\ M. Matricardi ${ }^{(10)}$, H. Revercomb ${ }^{(11)}$, C. Serio ${ }^{(12)}$, L. Strow ${ }^{(13)}$, \\ P. Schlüssel ${ }^{(14)}$, D. Klaes $^{(14)}$, C. Larigauderie ${ }^{(1)}$ \\ (1) Centre national d'études spatiales (Cnes) \\ (2) UK Met Office \\ (3) Laboratoire de physique moléculaire pour l'atmosphère et l'astrophysique \\ (LPMAA) \\ (4) Laboratoire atmosphères, milieux, observations spatiales (LATMOS) \\ (5) Université libre de Bruxelles (ULB) \\ (6) Institut Pierre-Simon Laplace/Laboratoire de météorologie dynamique \\ (IPSL/LMD) \\ (7) National Center for Atmospheric Research (NCAR) \\ (8) National Oceanic and Atmospheric Administration/ \\ Satellite Applications and Research (NOAA/STAR) \\ (9) Météo-France \\ (10) European Centre for Medium-Range Weather Forecast (ECMWF) \\ (11) Cooperative Institute for Meteorological Satellite Studies (CIMSS), \\ université de Madison \\ (12) Université de la Basilicate \\ (13) Université de Maryland \\ (14) European Organisation for the Exploitation of Meteorological Satellites \\ (Eumetsat)
}

asi (Infrared Atmospheric Sounding Interferometer) est un interféromètre de Michelson couvrant le domaine spectral infrarouge $645-2760 \mathrm{~cm}^{-1}(3,8$ à $15,5 \mu \mathrm{m})$, destiné au sondage atmosphérique à haute résolution. L'objectif affiché est d'une précision de $1 \mathrm{~K}$ par tranche de $1 \mathrm{~km}$ pour la température et de $10 \% / \mathrm{km}$ pour l'humidité. Il s'agit d'un capteur hyperspectral ${ }^{(1)}$, qui présente 8461 canaux quand la plupart des instruments n'en disposent que de quelques-uns et dont la résolution spectrale permet d'analyser finement les spectres des molécules actives dans l'infrarouge, autorisant la détection de gaz traces et la restitution des profils atmosphériques avec une bonne résolution verticale. Réellement innovant, cet instrument a été conçu par le Centre national d'études spatiales (Cnes), voir Cayla, 2001. Il a été réalisé et financé conjointement par le Cnes et l'Organisation européenne pour l'exploitation des satellites météorologiques (Eumetsat) et développé par
Thales Alenia Space (Blumstein et al., 2004 ; Siméoni et al., 2004). Le premier modèle de vol a été embarqué sur le satellite Metop-A, lancé le 22 octobre 2006. Deux autres modèles sont déjà assemblés et voleront sur Metop- $B$ (2012) et Metop-C (2016). Ces satellites, qui sont mis en œuvre par Eumetsat, constituent la contribution européenne à l'International Joint Polar System, Metop assurant la couverture du matin (heure du nœud descendant : 9 h 30) complémentaire de celle des satellites météorologiques polaires américains.

Iasi fournit des mesures de luminance infrarouge (radiances) dans 8461 canaux spectraux sur des pixels de $12 \mathrm{~km}$ de diamètre (voir encadré p. 21).

(1) Se dit d'un instrument doté d'au moins une centaine de canaux spectraux. Ces instruments sont en général des spectromètres ou des interféromètres. 


\section{Abstract}

The exceptional performance of IASI, METOP's multispectral atmospheric sounder

The second international IASI conference took place in Sévrier (France) from 24 to 29 January 2010. About 150 scientists from all countries attended the conference to assess the IASI performance, discuss the processing of the data, examine the main results of its exploitation since its launch three years ago and also to consider developments. In this article we describe the IASI instrument embarked onboard Eumetsat's three Metop satellites series aloft until 2020. The extraordinary performance of this instrument, which is now considered as a reference for infrared sensors' calibration is shown. The data processing scheme is recalled and the perspectives of upgrading are explained. The conference was presented with the most recent results in the various application domains of IASI, numerical weather forecasting, atmospheric composition and climate monitoring, which are emphasized. Finally, the focus is on the future with the expected evolution of the current products and also the perspectives after the IASI era with the sounder on Meteosat Third Generation and also the IASI New Generation, IASI-NG.

(1) Airs : Atmospheric Infrared Sounder, spectromètre à réseaux de la National Aeronautics and Space Administration (Nasa), embarqué sur le satellite Aqua.

(2) AATSR : Advanced Along Track Scanning Radiometer.

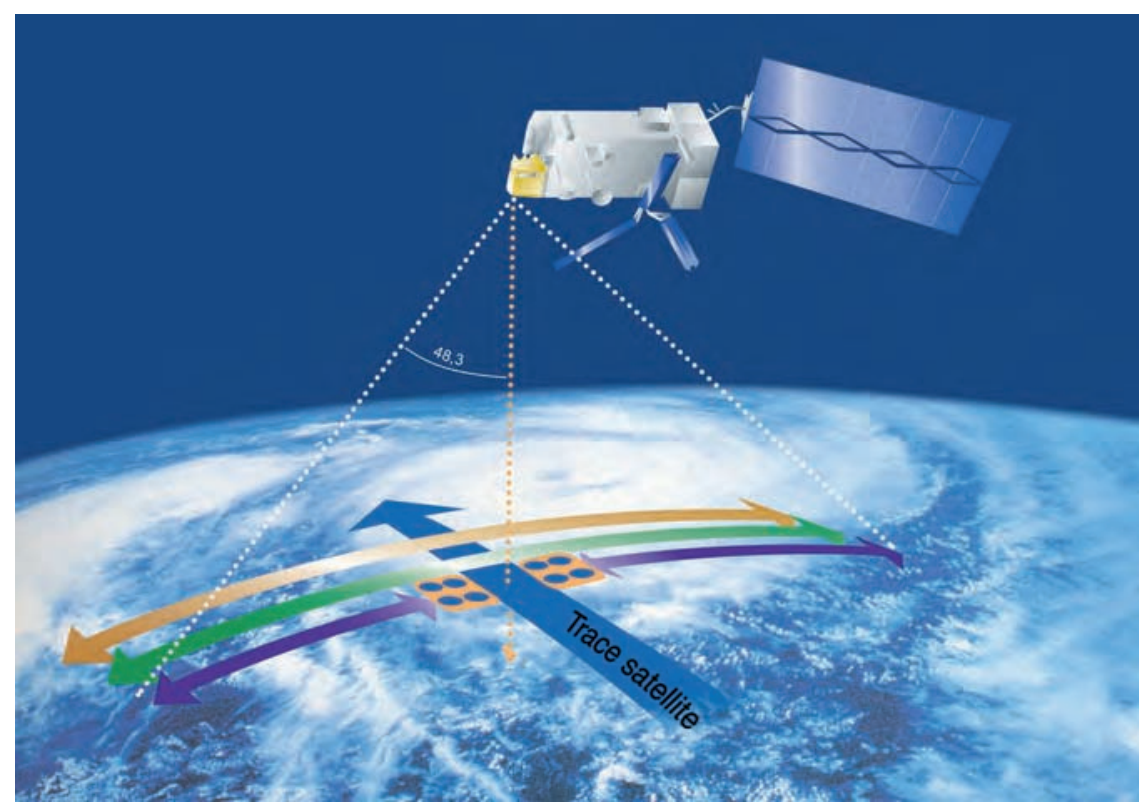

Figure 1 - Géométrie de visée de l'instrument lasi.

Un balayage de part et d'autre de la trace du satellite permet de couvrir une bande de $2100 \mathrm{~km}$ de large (figure 1).

L'étalonnage radiométrique est d'une précision remarquable ainsi que le confirme l'interétalonnage avec le spectromètre Airs ${ }^{(1)}$ réalisé soit aux points de croisement orbitaux (figure 2), soit par double différence (Strow et al., 2010) ou par comparaison avec des mesures de l'imageur AATSR ${ }^{(2)}$ (Remedios et al., 2010). La contamination par la glace sur la fenêtre d'entrée de la boîte froide, où sont logés les détecteurs refroidis jusqu'à $91 \mathrm{~K}$, est suivie avec précision et parfaitement maîtrisée. Elle s'avère faible et ne requerra qu'un nombre réduit de décontaminations. Les données relevées régulièrement par le centre d'expertise technique (TEC) du
Cnes au cours de la recette en vol (Blumstein et al., 2007) et, depuis lors, en phase de routine (Buffet et al., 2010) ou le suivi des radiances ( L1 monitoring ») réalisé par Eumetsat (Fiedler et al., 2010) confirment l'extrême stabilité temporelle de Iasi aux plans radiométrique et spectral. La stabilité par rapport à Airs est illustrée en figure 3 (Tobin et al., 2010). La précision, la stabilité et la couverture continue du spectre dans l'infrarouge thermique confèrent à l'instrument Iasi un rôle d'étalon pour plusieurs capteurs infrarouges tels que les radiomètres imageurs très utilisés, embarqués sur les satellites météorologiques opérationnels Météosat ou des satellites américains. Cet interétalonnage est essentiel pour le suivi des variables climatiques. Les méthodes appropriées sont étudiées et
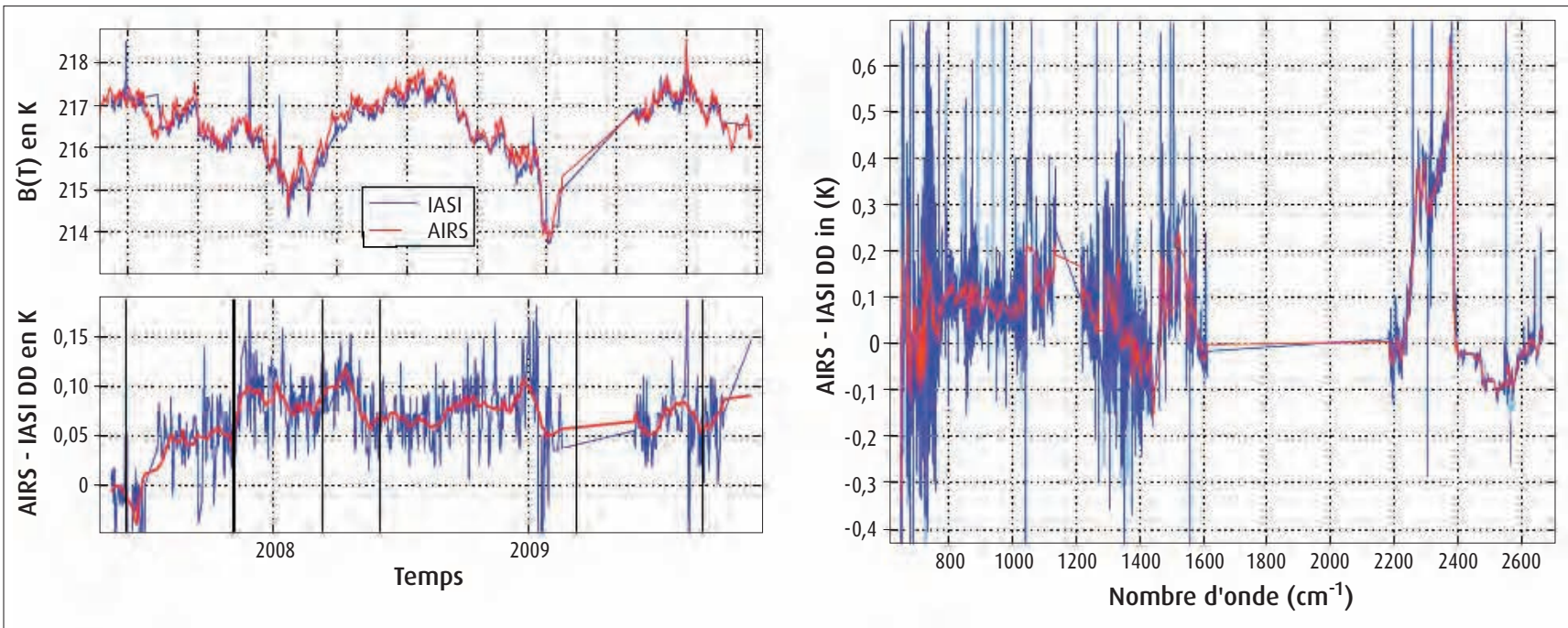

Figure 2 - Interétalonnage de lasi avec Airs aux points de croisement orbitaux. Cette figure montre, en haut à gauche, l'évolution des luminances de lasi et Airs à $698 \mathrm{~cm}{ }^{-1}$ et en bas à gauche, l'écart entre les deux observations calculé selon la technique de Double différence entre observation et simulation par un modèle : Obs-Calc (Airs) - ObsCalc (lasi). À droite, la courbe rouge représente l'écart moyen en fonction du nombre d'onde. (C L. Strow) 
mises en place dans le cadre du Global Satellite Inter-Calibration System (GSICS, http://gsics.wmo.int) [Goldberg et al., 2011].

Les interruptions dues aux impacts par des ions lourds ou provoquées par la perte de référence pour le calage du zéro de la différence de marche optique des interférogrammes sont rares et des solutions ont été mises en place pour réduire la durée d'interruption, circonvenir les incidents au-dessus de la zone d'anomalie géomagnétique du Pacifique sud, etc. Au total, la disponibilité de Iasi approche $96 \%$. Des modifications du logiciel de prétraitement conçu au Cnes ont été intégrées récemment afin d'augmenter encore la disponibilité des données effectivement utilisables pour les applications opérationnelles ou scientifiques.

Eumetsat réceptionne les données globales à la station de Svalbard, les traite puis les dissémine dans un délai de 2 h 15 après l'acquisition. La transmission en temps quasi réel des volumes gigantesques de données $(\sim 1,3$ million de spectres par jour) est réalisée via le système Eumetcast, qui s'appuie sur des satellites de télévision. Pour les pays non couverts, une sélection de 366 canaux proposée par Collard (2007) est distribuée via le Système mondial de télécommunications de l'Organisation météorologique mondiale (OMM). Le flot de données inclut les produits de niveau 1 (spectres en radiance étalonnés et géolocalisés) et de niveau 2 (produits géophysiques). À brève échéance, au « day- 2 », les données fournies incluront également des spectres fortement compressés selon une méthode statistique établie par Eumetsat (Hultberg, 2010). En France,

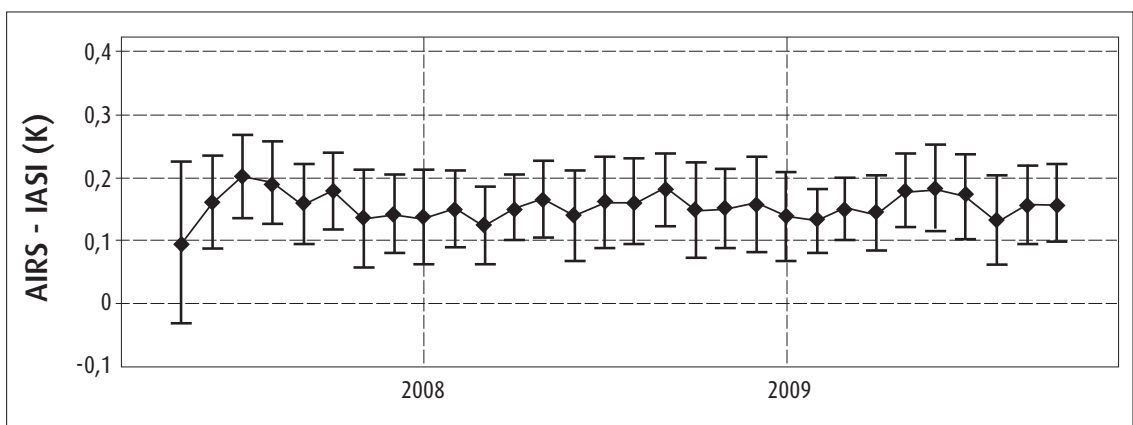

Figure 3 - Stabilité de l'instrument : comparaison des moyennes mensuelles de lasi et Airs aux points d'intersection des orbites. Les données sont comparées pour la bande de 1460 à $1527 \mathrm{~cm}^{-1}$. (C) D. Tobin) les données sont reçues via Eumetcast à Toulouse, à Lannion et à l'Institut PierreSimon Laplace (IPSL) pour le pôle de compétence en chimie atmosphérique Ether.

\section{Utilisation des données}

Les données de niveau 1 sont utilisées de façon opérationnelle par les centres de prévision météorologique qui les assimilent directement dans les modèles.

Pour produire des données géophysiques, Eumetsat a développé des traitements de niveau 2, paramétrables et configurables, s'inspirant des algorithmes proposés par l'ISSWG ${ }^{(1)}$ (Schlüssel et al., 2005). Ces traitements permettent de retrouver dans chaque pixel des profils atmosphériques de température et d'humidité sur 100 niveaux, des profils et colonnes d'ozone, les caractéristiques de la couverture nuageuse, des colonnes de $\mathrm{CO}$, de $\mathrm{CH}_{4}$, de $\mathrm{CO}_{2}$ et de $\mathrm{N}_{2} \mathrm{O}$. Les profils

\section{Les données lasi}

Après un traitement complet de la télémesure, les données de niveau 1c de lasi consistent en des spectres de luminance, bien étalonnés spectralement et radiométriquement, échantillonnés avec un pas spectral régulier et proprement géoréférencés. Les luminances sont fournies dans 8461 canaux tous les $0,25 \mathrm{~cm}^{-1}$ après apodisation (opération classique pour les mesures de spectres par transformée de Fourier, qui permet d'homogénéiser la fonction spectrale de l'instrument sur tout le domaine spectral) par une fonction de réponse instrumentale unique d'une largeur à mi-hauteur de $0,5 \mathrm{~cm}^{-1}$. Les produits 1c incluent également des informations dérivées d'AVHRR (Advanced Very High Resolution Radiometer) permettant de caractériser les surfaces présentes à l'intérieur des pixels de $12 \mathrm{~km}$ de diamètre au nadir.

Les pixels de lasi sont les éléments d'une matrice $2 \times 2$ espacés de $18 \mathrm{~km}$. Une « boîte » de $2 \times 2$ pixels est coregistrée avec la position d'un pixel Amsu (Advanced Microwave Sounder Unit) de $50 \mathrm{~km}$ de diamètre. Le balayage de $-48,3$ à $+48,3^{\circ}$ de part et d'autre de la trace (soit une fauchée au sol de $2100 \mathrm{~km}$ ) est réalisé en 8 secondes par un miroir à compensation de filé (dispositif mécanique pour maintenir le pointage sur un pixel pendant l'avance du satellite). II y a au total 30 positions de visée et 3 visées d'étalonnage. Les spectres de luminance sont étalonnés radiométriquement par visée d'un corps noir et par celle de l'espace froid. Une calibration spectrale d'une très grande précision est également réalisée (précision relative d'environ $2 \mathrm{ppm}$ ). atmosphériques et les informations sur les nuages ont été validés et, leur conformité aux spécifications des utilisateurs ayant été établie, sont distribués « en opérationnel » par Eumetsat. En chimie atmosphérique, les produits ozone et $\mathrm{CO}$ ont été validés (Boynard et al., 2009 ; George et al., 2009).

Aux États-Unis, la National Oceanic and Atmosphéric Administration (NOAA) a développé ses propres traitements de niveau 2 (Gambacorta, communication privée).

De nombreux laboratoires de recherche ont également mis au point des algorithmes pour restituer leurs propres produits. De façon générale, à la différence des centres de prévision, ils utilisent tous les pixels et un nombre élevé de canaux choisis de façon spécifique parmi les 8461 éléments spectraux de Iasi pour chaque espèce ou paramètre cible.

Les progrès dans le traitement des données et la qualité des produits sont suivis par l'ISSWG, établi depuis 1996 par le Cnes et Eumetsat, qui identifie les domaines prioritaires, met en place des exercices d'intercomparaison et fait des recommandations aux agences en charge du sondeur.

\section{L'apport à la prévision météorologique}

Après le lancement de Metop, les données Iasi ont été très vite intégrées dans les schémas d'assimilation des modèles globaux et leur impact positif a été rapidement démontré. L'utilisation des données Iasi est désormais très fréquente dans les modèles globaux. Peu à

(1) ISSWG : International IASI Sounding dont sont membres tous les auteurs de cette communication. Scientific Working Group, groupe scientifique 
peu, l'utilisation des données a été étendue aux modèles à aire limitée (LAM). Les développements les plus récents concernent l'intégration dans les modèles régionaux à courte échéance. Pour chaque modèle, les conditions d'utilisation des données diffèrent. Une classification a été établie par Hilton (2010) et est reproduite dans le tableau 1.

\section{Modèles globaux et modèles à aire limitée}

Parmi les centres qui assimilent les données Iasi, on compte notamment :

- le Centre européen pour les prévisions météorologiques à moyen terme (CEPMMT ou ECMWF en anglais) ;

- Météo-France ;

- le UK Met Office, service météorologique national britannique ;

- le Deutscher Wetterdienst (DWD), service météorologique national allemand ;

- le Met No, service météorologique norvégien ;

- la National Oceanic and Atmosphéric Administration (NOAA) ;

- le National Centers for Environmental Prediction (NCEP, États-Unis) ;

- le Naval Research Laboratory (NRL) ;

- Environnement Canada.
Tous assimilent des radiances, sauf le DWD qui, pour son modèle à aire limité, utilise un schéma de «nud$\operatorname{ging}^{(1)} »$. Les données sont largement écrémées, les centres n'utilisant qu'un pixel tous les $120 \mathrm{~km}$ environ. Cet écrémage ( "thinning ») est effectué pour éviter d'introduire des erreurs corrélées dans l'assimilation. De plus, les centres n'utilisent pour l'instant qu'un nombre assez réduit de canaux (200 au plus), la plupart dans les bandes du $\mathrm{CO}_{2}$ aux grandes longueurs d'onde. En effet, ce domaine d'absorption du gaz carbonique a été très utilisé (mais avec une résolution spectrale nettement moins bonne) et est donc le mieux connu (en particulier pour la spectroscopie) et c'est donc avec l'utilisation des canaux dans cette région spectrale que l'apport de Iasi est attendu en premier lieu pour le sondage de température. Seuls certains centres commencent à utiliser une dizaine de canaux des bandes intenses d'absorption de la vapeur d'eau, en ne retenant que ceux qui ont des fonctions poids au-dessus du niveau $700 \mathrm{hPa}$. Le nombre de canaux sélectionnés est souvent restreint au-dessus des terres émergées et des glaces. Il est fortement dépendant des contrôles de qualité mis en place pour rejeter l'information incompatible avec l'erreur a priori du modèle. La limite supérieure d'altitude des modèles (en particulier pour les LAM) empêche l'utilisation des canaux sensibles à haute altitude. Pour les corrections de biais, deux méthodes sont en concurrence. Le code de transfert radiatif utilisé par la plupart des centres est le code RTTOV ${ }^{(2)}$, toutefois les centres américains préfèrent le code $\mathrm{CRTM}^{(3)}$. Les erreurs d'observation prises en compte dans l'assimilation dominent largement la somme du bruit instrumental et de l'incertitude de transfert radiatif (figure 4), ce qui tendrait à prouver que beaucoup peut encore être fait pour améliorer l'utilisation des données du sondeur Iasi.

Si, pour l'instant, les centres limitent l'utilisation des radiances Iasi aux cas de ciel clair ou à une utilisation restreinte des canaux dont le maximum de sensibilité se

(1) Le nudging (ou relaxation newtonienne) consiste à ajouter aux équations d'état du système un terme de rappel, proportionnel à la différence entre les observations et la quantité correspondante calculée par la résolution du système des équations d'état. Le modèle apparaît alors comme une contrainte faible et le terme de rappel force les variables du modèle à coller avec les observations. (2) RTTOV : Code de transfert radiatif rapide pour la simulation des mesures des satellites météorologiques.

(3) CRTM : Community Radiative Transfer Model, modèle rapide de transfert radiatif.

Tableau 1 - Utilisation des données lasi dans les modèles de prévision numérique dans différents services météorologiques (Hilton et al., 2010). En rouge, les modèles avec assimilation opérationnelle, en noir les modèles avec assimilation en test. (Crédit : F. Hilton)

\begin{tabular}{|c|c|c|c|c|c|c|}
\hline & Modèle & Domaine & $\begin{array}{l}\text { z/P, nombre } \\
\text { de niveaux }\end{array}$ & $\begin{array}{l}\text { Résolution } \\
\text { horizontale }\end{array}$ & $\begin{array}{l}\text { Type } \\
\text { d'assimilation }\end{array}$ & $\begin{array}{l}\text { Correction } \\
\text { des biais }\end{array}$ \\
\hline \multirow[t]{2}{*}{ Met Office } & $\begin{array}{l}\text { Global } \\
\text { NAE }\end{array}$ & $\begin{array}{l}\text { Global } \\
\text { Atlantique nord } \\
\text { et Europe }\end{array}$ & $80 \mathrm{~km} / \mathrm{L70}$ & $\begin{array}{l}\sim 60 \mathrm{~km} \\
\sim 12 \mathrm{~km}\end{array}$ & $\begin{array}{l}\text { 4D-Var } \\
\text { 4D-Var }\end{array}$ & $\begin{array}{l}\text { Harris\&Kelly } \\
\text { Harris\&Kelly }\end{array}$ \\
\hline & UK4/UKVD* & UK & 40 km/L70 & 4/1,5 km & 3 ou 4D-Var & Harris\&Kelly \\
\hline Météo-France & $\begin{array}{l}\text { Arpège } \\
\text { Aladin } \\
\text { Arome }\end{array}$ & $\begin{array}{l}\text { Global } \\
\text { Europe de l'Ouest } \\
\text { France }\end{array}$ & $\begin{array}{l}0,1 \mathrm{hPa} / \mathrm{L60} \\
0,1 \mathrm{hPa} / \mathrm{L70} \\
0,1 \mathrm{hPa} / \mathrm{L60}\end{array}$ & $\begin{array}{l}10-60 \mathrm{~km} \\
7,5 \mathrm{~km} \\
2,5 \mathrm{~km}\end{array}$ & $\begin{array}{l}\text { 4D-Var } \\
\text { 3D-Var } \\
\text { 3D-Var }\end{array}$ & $\begin{array}{l}\text { VarBC*** } \\
\text { VarBC } \\
\text { VarBC }\end{array}$ \\
\hline ECMWF & Global & Global & 80 km/L91 & $\sim 25 \mathrm{~km}$ & 4D-Var & VarBC \\
\hline DWD & $\begin{array}{l}\text { GME } \\
\text { Cosmo-EU }\end{array}$ & $\begin{array}{l}\text { Global } \\
\text { Europe }\end{array}$ & $\begin{array}{l}10 \mathrm{hPa} / \mathrm{L60} \\
20 \mathrm{hPa} / \mathrm{L} 40\end{array}$ & $\begin{array}{l}40 \mathrm{~km} \\
7 \mathrm{~km}\end{array}$ & $\begin{array}{l}\text { 3D-Var } \\
\text { Nudging }\end{array}$ & $\begin{array}{l}\text { Harris\&Kelly } \\
\text { Harris\&Kelly }\end{array}$ \\
\hline Met No & Harmonie & $\begin{array}{l}\text { Pôle Nord } \\
\text { et Europe }\end{array}$ & $0,2 \mathrm{hPa} / \mathrm{L60}$ & $11-16$ km & 3D-Var & VarBC \\
\hline NCEP & $\begin{array}{l}\text { GFS } \\
\text { NAM }\end{array}$ & $\begin{array}{l}\text { Global } \\
\text { Régional }\end{array}$ & $\begin{array}{l}0,27 \mathrm{hPa} / \mathrm{L} 64 \\
2 \mathrm{hPa} / \mathrm{L60}\end{array}$ & $\begin{array}{l}\sim 35 \mathrm{~km} \\
12 \mathrm{~km}\end{array}$ & $\begin{array}{l}\text { 3D-Var } \\
\text { 3D-Var }\end{array}$ & $\begin{array}{l}\text { VarBC } \\
\text { VarBC }\end{array}$ \\
\hline Env. Canada & GEM & Global & $0,1 \mathrm{hPa} / \mathrm{L} 80$ & $\sim 33 \mathrm{~km}$ & 4D-Var & $\begin{array}{l}\text { Self udpadting } \\
\text { H\&́K like }\end{array}$ \\
\hline NRL & NAVDAS-AR & Global & $0,4 \mathrm{hPa} / \mathrm{L} 42$ & $\sim 55 \mathrm{~km}$ & 4D-Var & Harris\&Kelly \\
\hline
\end{tabular}

* ECMWF : European Center for Medium range Weather Forecast.

** Schéma variationnel pour correction des biais des mesures vis-à-vis des champs des modèles de prévision. 


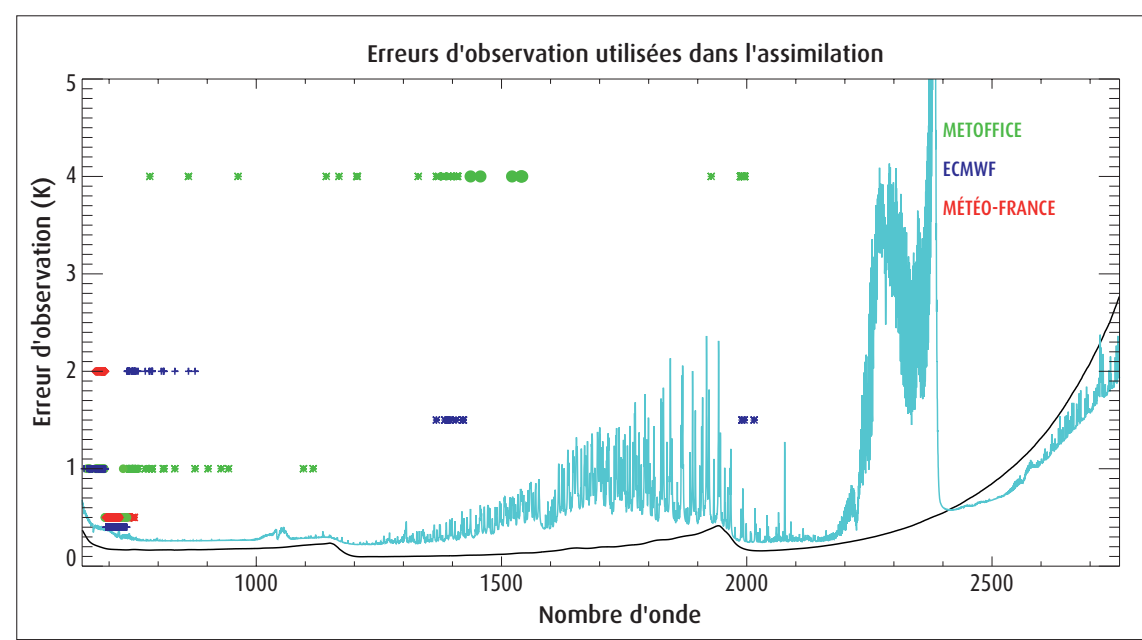

Figure 4 - Erreurs d'observation utilisées dans l'assimilation par différents centres d'assimilation : en noir, bruit radiométrique et, en cyan, incertitude totale (bruit radiométrique + erreur de transfert radiatif). Cette figure illus tre le fait que les erreurs d'observation prises en compte dans l'assimilation excèdent notablement l'incertitude réelle des observations. (C F. Hilton)

situe au-dessus des nuages (déterminés suivant des méthodes différentes d'un centre à l'autre), la tendance est à aller vers une utilisation des radiances affectées par les nuages. Les premières réflexions sur ce sujet laissent espérer des progrès

significatifs dans la description de l'atmosphère dans les régions sensibles (McNally, 2010).

L'impact positif des données Iasi sur les scores de prévision est admis par

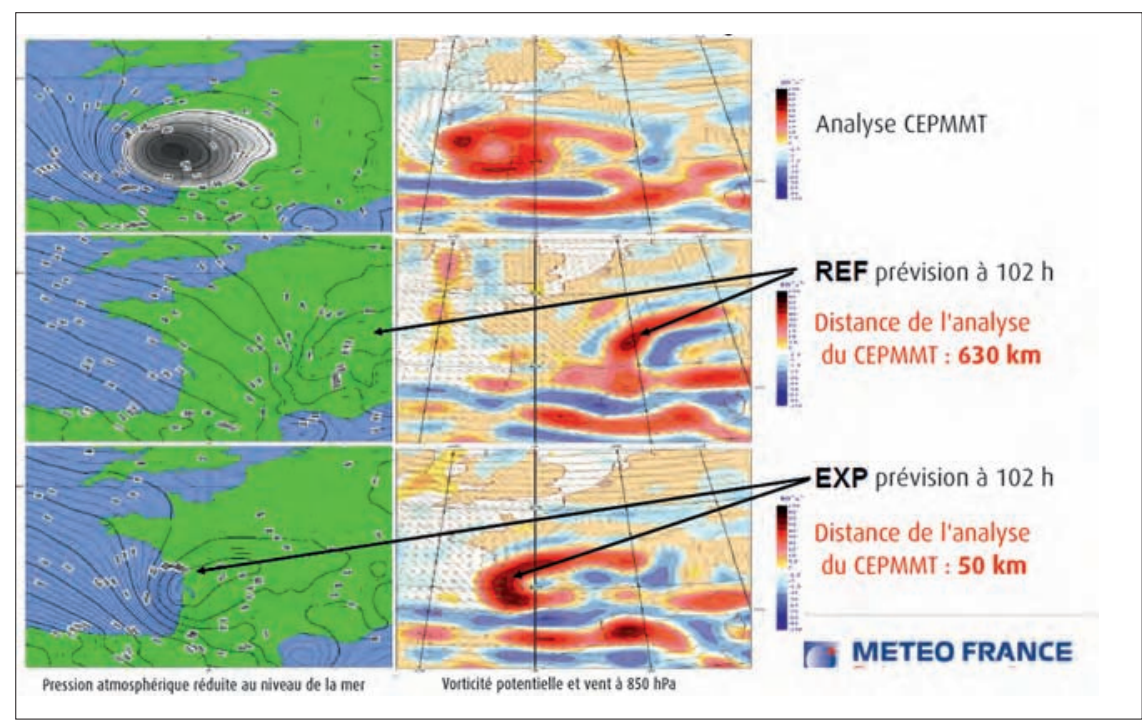

Figure 5 - Impact de l'utilisation des radiances nuageuses lasi dans le cas de la tempête Klaus (Fourrié, 2010). Les cartes représentent le champ de pression (à gauche) et le vent et la vorticité potentielle à $850 \mathrm{hPa}$ (à droite). La première ligne montre l'analyse du CEPMMT le 24/01/2009 à 06 UTC. La deuxième ligne est la prévision Arpège à 102 heures pour cette échéance obtenue sans utiliser lasi (REF). En bas (EXP), en utilisant les données lasi dans la prévision. La comparaison montre que le champ prévu lorsque les données lasi sont utilisées est bien plus proche de la réalité (l'analyse). [@ N. Fourrié] tous les centres. Mais les méthodes d'évaluation de l'impact étant différentes d'un centre à l'autre, une comparaison directe est difficile. Chaque centre exprime ses résultats à travers divers graphiques dont un exemple est donné en figure 5. Limpact positif de Iasi est vraiment bon aux échéances moyennes (72 heures et un peu plus). Comme attendu l'impact est plus fort dans l'hémisphère sud, mais il y a également un bénéfice dans l'hémisphère nord. L'assimilation des données Iasi apporte un impact de même qualité, voire meilleur, que les autres systèmes spatiaux antérieurs (comme le radiomètre micro-ondes $\left.\mathrm{Amsu}^{(1)}\right)$. L'impact de Iasi semble légèrement supérieur à celui d'Airs (figure 6), l'addition des deux permettant un gain appréciable par rapport à chacun d'eux. Une expérience de «privation» (conséquence pour la prévision de la suppression d'une observation) des données des différents instruments de Metop a montré également le gain lié à la synergie de l'ensemble des mesures (Bauer et al., 2009, communication privée).

L'utilisation des données Iasi dans des modèles à domaines limités pose quelques problèmes spécifiques :

- les domaines concernent des régions continentales où la connaissance de l'émissivité des sols est indispensable ; - les corrections de biais, généralement établies à l'échelle globale, demandent donc une attention particulière. D'ailleurs, les corrections des modèles globaux ne sont pas toujours applicables telles quelles. Ce problème est bien cerné et les centres de prévision explorent différentes méthodes, y compris celle de redéfinir une correction de biais spécifique. Mais il faut être prudent car les pièges sont nombreux : grandes variations saisonnières, échantillonnage de tous les angles de visée, etc. ;

(1) Amsu : Advanced Microwave Sounding Unit radiomètre à balayage opérant dans le domaine des micro-ondes.

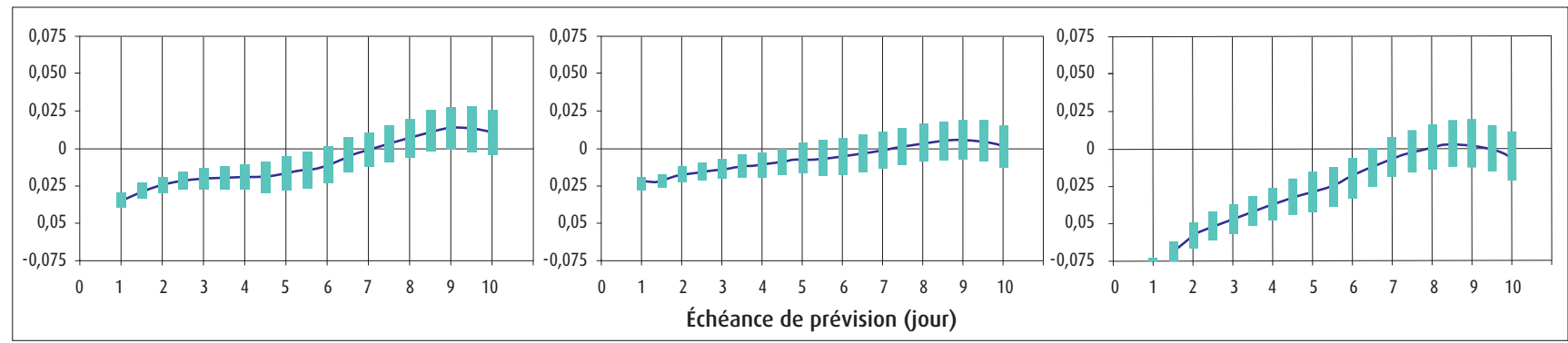

Figure 6 - Apport des spectromètres infrarouges à la prévision numérique dans l'hémisphère sud. L'ordonnée représente la différence de corrélation d'anomalie à 500 hPa entre une prévision avec assimilation des données de lasi (resp. Airs, resp. lasi + Airs) et une prévision de référence n'assimilant pas ces données. Les impacts positifs sont indiqués par les valeurs négatives. Avec lasi, cet impact se fait sentir jusqu'à 6 jours. L'utilisation des deux sondeurs apporte une amélioration significative du score (jusqu'à 7 jours). 
- la nécessité d'estimer la température stratosphérique.

En dépit de ces difficultés, les résultats obtenus sont prometteurs. En témoigne une illustration de l'expérience d'évaluation menée sur la situation de la tempête Klaus (figure 5).

\section{Modèles à mésoéchelle}

L'utilisation des données Iasi dans les modèles à mésoéchelle tels qu'Arome (Météo-France) ou UKVD (Met Office) apparaît prometteuse. Nombre d'observations satisfont au test de qualité et peuvent être utilisées avec un impact visible sur l'analyse. L'utilisation de Iasi a un impact sur le champ de vent et sur les précipitations (figure 7) prévues par le modèle, identique à celui résultant de l'assimilation des radars de précipitions, avec un très léger gain sur les prévisions de précipitations (Guidard, 2010). Pour Arome, des résultats encourageants ont été obtenus avec un écrémage moins lâche $(80 \mathrm{~km}$ au lieu de $120 \mathrm{~km})$.

\section{Domaines de progrès}

Des études sont en cours pour assimiler davantage de données Iasi :

- utiliser les radiances en situation nuageuse ;

- assimiler plus de canaux vapeur d'eau ;

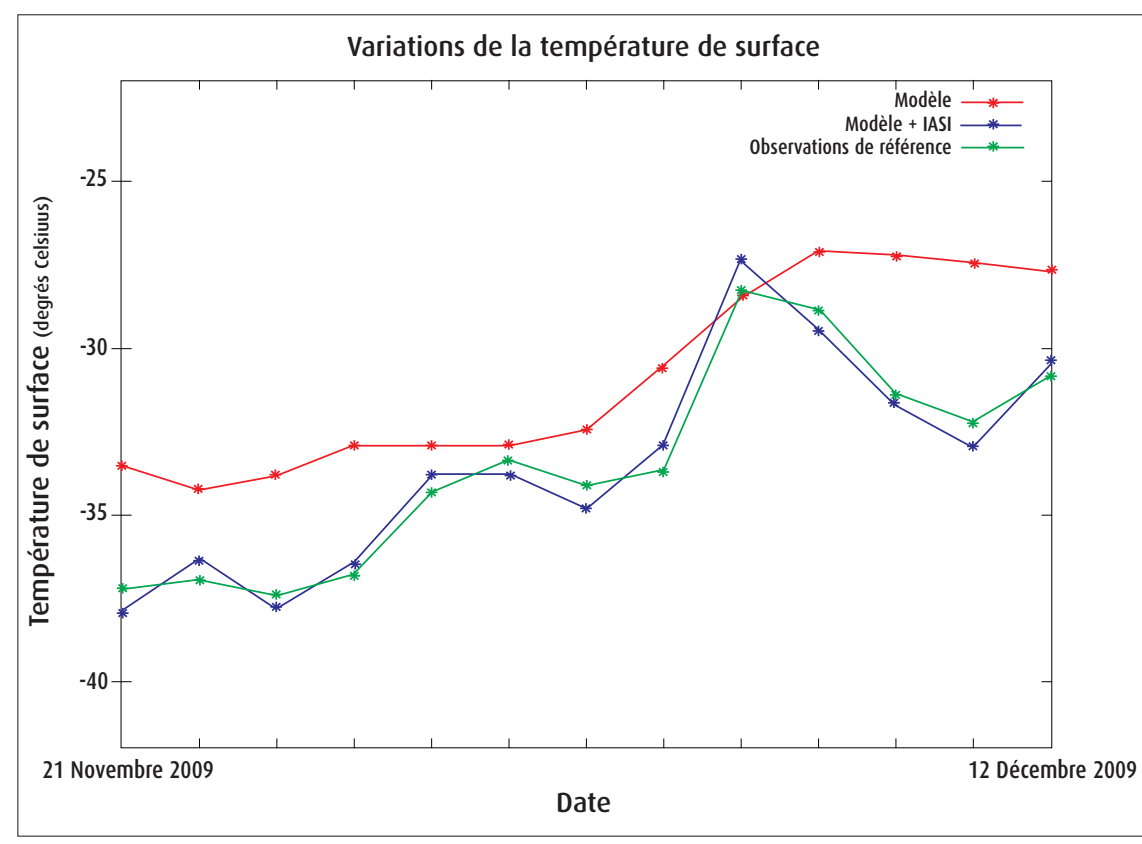

Figure 8 - Comparaison des températures de surface obtenues avec Arome intégrant lasi (en bleu), sans lasi (en rouge) et mesurées au sol à Concordia en Antarctique (en noir). [C A. Vincensini et F. Rabier]

- assimiler plus de radiances sur les terres émergées et les glaces (en caractérisant mieux la température et l'émissivité de surface) ;

- assimiler les aérosols, les espèces chimiques et les gaz traces déduits des mesures de Iasi.

Des investigations sont menées pour mieux décrire et comprendre les corrélations entre les erreurs d'observation des différents canaux, puis pour les utiliser dans l'assimilation dans les

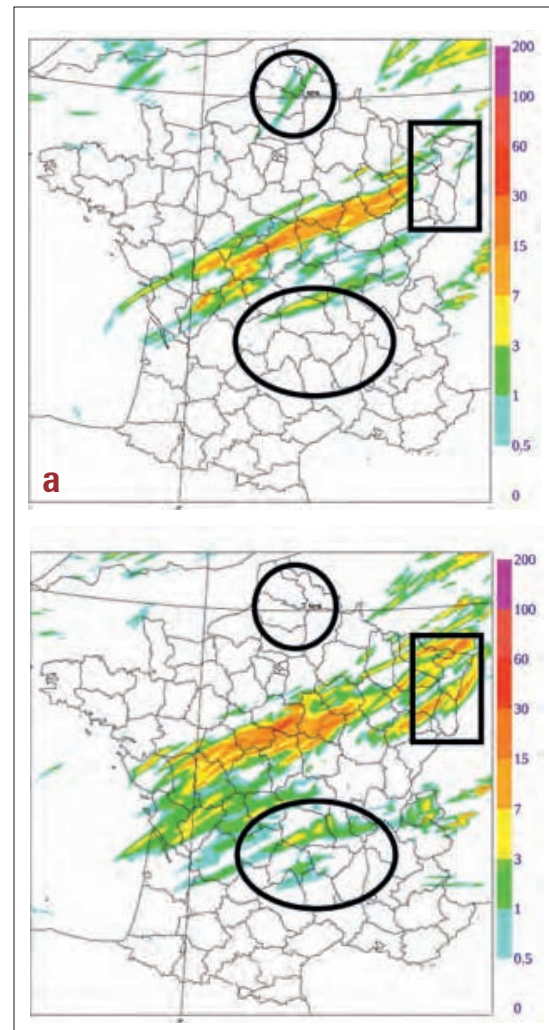

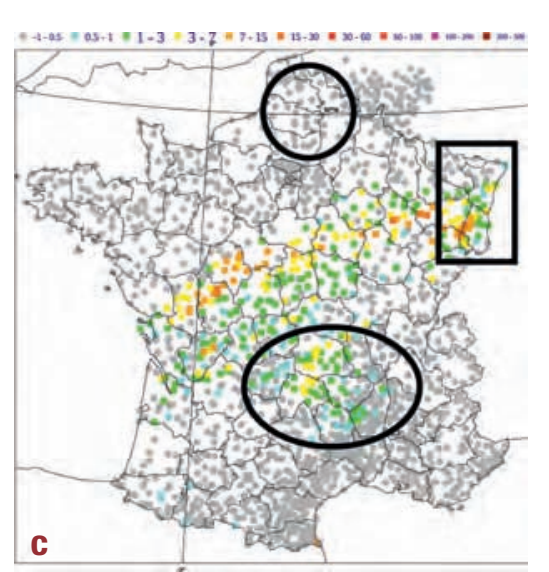

Figure 7 - Comparaison des prévisions de précipitation du modèle Arome, observations déduites de lasi et mesures pluviométriques. Le 21 mai 2009, entre $00 \mathrm{~h}$ UTC et $12 \mathrm{~h}$ UTC

a) prévision à 12 heures sans I'utilisation de lasi ; b) prévision à 12 heures avec utilisation de lasi ; c) les mesures pluviométriques.

On observe dans le Nord de la France l'absence de pluie mesurée conformément à la prévision " lasi » et en Alsace et sur le Massif central des pluies bien prévues avec lasi. (@ V. Guidard) modèles de prévision numérique du temps.

L'expérience Concordiasi (Rabier, 2010) avait pour but de tester ces possibilités de mieux utiliser les données Iasi dans les modèles d'assimilation, en particulier audessus des glaces de l'Antarctique. Cette expérience a déjà produit nombre de résultats scientifiques de grand intérêt et l'exploitation des données se poursuit. À titre d'exemple, la figure 8 montre la bonne cohérence entre la température de surface analysée par le modèle Arome avec Iasi et la température de surface mesurée à Concordia en Antarctique.

En conclusion, de très bons résultats ont déjà été obtenus, mais l'utilisation d'un nouveau sondeur spatial très performant comme Iasi en prévision numérique nécessite de très longs développements qui se poursuivent pas à pas.

\section{La composition atmosphérique}

Le domaine spectral de Iasi couvre des bandes d'absorption intenses des gaz à effet de serre tels que $\mathrm{CO}_{2}, \mathrm{CH}_{4}, \mathrm{~N}_{2} \mathrm{O}$ ou $\mathrm{O}_{3}$, une bande de $\mathrm{CO}$ et une multitude de bandes des molécules présentes de façon naturelle à l'état de trace dans l'atmosphère ou résultant de l'action anthropique tels que les composés organiques volatils ou les chlorofluorocarbones. Iasi s'avère donc un outil très précieux pour l'analyse de la composition atmosphérique avec pour finalité le 
suivi opérationnel de la qualité de l'air, la détection des impacts accidentels (éruptions volcaniques, feux) ou dus à l'action anthropique (feux de brousse) et l'étude des interactions chimie-climat. Iasi est sans doute le premier sondeur atmosphérique infrarouge qui permette, grâce à sa couverture continue de l'infrarouge thermique, avec une relativement bonne résolution spectrale et une très bonne qualité radiométrique, d'accéder à tant d'espèces. Si pour certaines espèces l'instrument n'autorise à ce stade que la détection, il en est que Iasi permet de quantifier avec une précision très intéressante et une résolution verticale qui, bien que limitée, s'avère utile à l'exploitation scientifique.

\section{Les espèces majeures}

\section{L'ozone}

Iasi permet d'aborder plusieurs problématiques scientifiques liées à l'ozone $\left(\mathrm{O}_{3}\right)$ :

- la surveillance de l'ozone stratosphérique et notamment du trou d'ozone en Antarctique ;

- le transport d'ozone et les intrusions vers la troposphère ;

- l'évolution de l'ozone troposphérique liée à la pollution atmosphérique et les relations entre la qualité de l'air et le climat (effet de serre dû à l'ozone troposphérique par exemple).

Une analyse du contenu en information montre que Iasi présente 2,5 à 3,5 degrés de liberté pour les profils d'ozone. Le nombre de degrés de liberté (Degrees of Freedom ou DOF suivant la terminologie de l'analyse en contenu d'information) donne une estimation du nombre d'éléments d'information indépendants en fonction de l'altitude. Il est donc possible non seulement de restituer le contenu intégré mais également trois colonnes partielles. La définition de ces colonnes diffère suivant les conditions géophysiques du lieu de l'observation. Le maximum de sensibilité est obtenu pour la haute troposphère (autour de $10 \mathrm{~km}$ ) et pour la très basse stratosphère autour de $18 \mathrm{~km}$. Iasi peut donc apporter des informations très utiles à l'étude des interactions troposphère-stratosphère (Upper Troposphere Lower

(1) Dans un problème d'inversion matricielle, la régularisation consiste à ajouter dans la fonction de coût à minimiser un terme quadratique dépendant de la variable à retrouver.

(2) MLS : Microwave Limb Sounder.

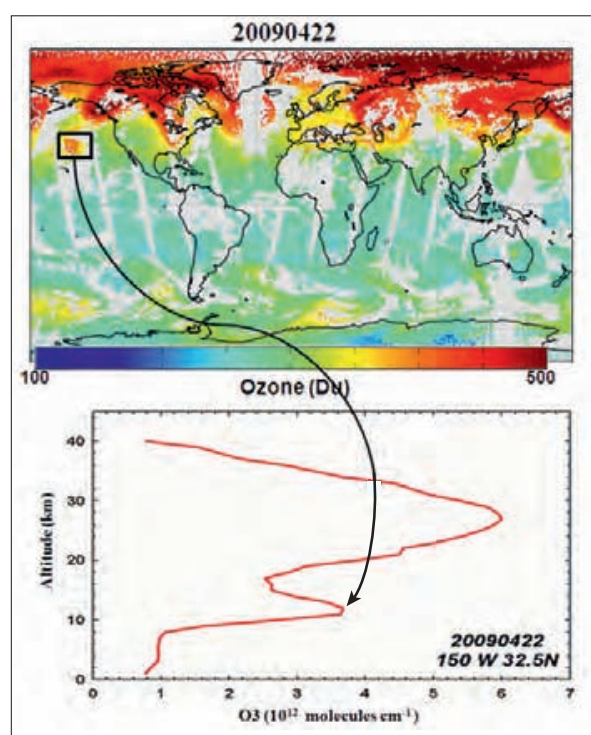

Figure 9 - Intrusion d'ozone stratosphérique vue par lasi le 22 avril 2009. De fortes concentrations (en orange) sont mesurées dans le Pacifique nord (voir encadré sur la figure du haut) pour la colonne totale d'ozone. Cette intrusion d'ozone stratosphérique descend jusque dans la troposphère comme le montre le profil de la figure du bas où un pic est mesuré vers 12 km. (C C. Scannell)

Stratosphere, UTLS). Il y a peu d'information au-dessus de $40 \mathrm{~km}$. Si le contraste thermique est favorable, les données Iasi sont relativement sensibles dans la basse troposphère (au-dessous de $6 \mathrm{~km}$ ) et, dans le cas de pollution assez sévère, Iasi peut être utilisé pour le suivi de la qualité de l'air. Les méthodes d'inversion utilisées sont soit des réseaux de neurones (August, 2010), soit des méthodes d'estimation optimale (Scannel et al., 2010 ; Barret, 2010) ou de régularisation ${ }^{(1)}$ (Eremenko et Dufour, 2010 ; Wassmann et Landgraf, 2010).

Les premières assimilations Iasi de l'ozone ont été réalisées et montrent des résultats plus qu'encourageants, que ce soit à partir des profils d'ozone (Massart, 2010) ou des radiances (Han et McNally, 2010). En effet, ces auteurs, qui comparent les profils d'ozone de Iasi avec ceux, très précis, du sondeur au limbe $\operatorname{MLS}^{(2)}$, montrent une précision au moins aussi bonne que celle des profils obtenus avec les sondeurs ozone classiques utilisant les bandes ultraviolettes (UV).

Une validation approfondie a été réalisée sur ces produits par croisement avec d'autres données satellite comme celles du spectromètre à balayage Gome-2 (Global Ozone Monitoring Experiment), de l'instrument OMI (Ozone Monitoring Instrument), du spectromètre imageur à balayage Sciamachy ou du sondeur au limbe MLS, aussi bien qu'avec des mesures in situ de sondes d'ozone (Barret, 2010). Typiquement, les biais sont de l'ordre de $6 \%$ pour la colonne totale (RMS de $7 \%$ ), avec une surestimation par rapport aux sondeurs UV. Ces biais sont en général assez réduits pour les colonnes partielles troposphériques (de l'ordre de $5 \%$ ) mais les écarts-types restent assez forts (environ $40 \%$ pour une colonne $0-6 \mathrm{~km}$; $20 \%$ pour une colonne $0-12 \mathrm{~km}$ et $10 \%$ pour une colonne 0-18 km). Pour les colonnes les plus basses, les erreurs proviennent essentiellement de la mauvaise connaissance des propriétés de surface (température et émissivité) et parfois des inversions de température au voisinage de la surface. Une part non négligeable incombe également aux nuages ou aux aérosols qui contaminent les mesures (Barret, 2010).

La production de cartes d'ozone est désormais opérationnelle à Eumetsat mais aussi dans certains laboratoires comme l'Université libre de Bruxelles (ULB). La production systématique de cartes permet le suivi dans le temps de phénomènes tels que les intrusions d'ozone stratosphérique dans la troposphère (figure 9). Les cartes d'ozone troposphérique sont aussi utilisées pour analyser la pollution des grandes mégapoles (figure 10 ; Dufour et al., 2010). Naturellement ces données viennent aussi compléter les

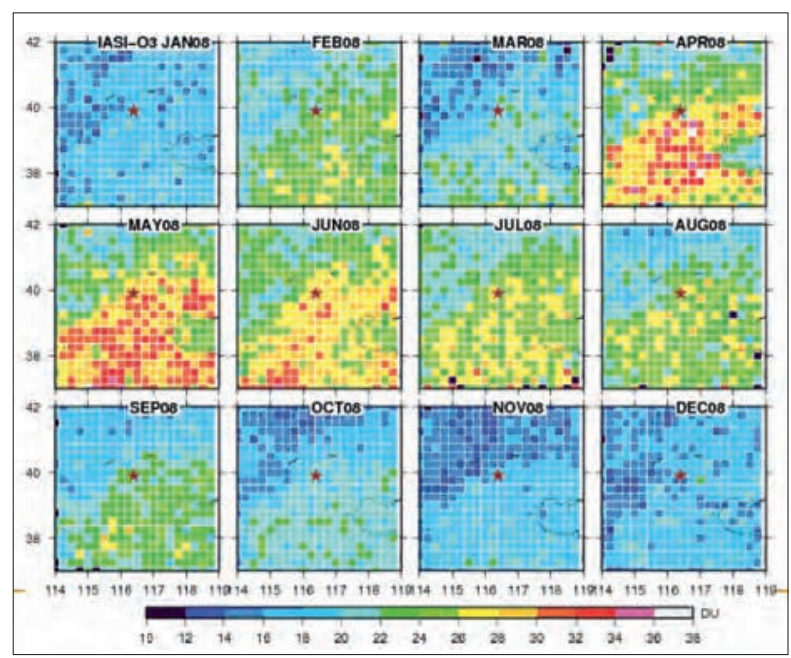

Figure 10 - Distribution spatiale mensuelle de l'ozone de la basse troposphère retrouvé avec lasi autour de Beijing (Chine). Les valeurs en rouge correspondent à de fortes concentrations. Celles-ci peuvent être observées à partir du printemps lorsque la photolyse devient importante dans les zones à forte concentration urbaine (Dufour et al, 2010). 
nombreuses données collectées pour analyser, année après année, le trou d'ozone de l'Antarctique. (Scannel et al., 2010)

\section{Le monoxyde de carbone}

Le monoxyde de carbone (CO) est un gaz réactif qui contribue à la formation des gaz à effet de serre $\mathrm{CO}_{2}$ et $\mathrm{O}_{3}$. Étant également un puits pour le radical $\mathrm{OH}$, sa présence influence la concentration de méthane dans l'atmosphère. C'est aussi un gaz que l'on retrouve dans les émissions résultant des processus de combustion (automobiles, centrales thermiques, foyers...) et en forte quantité dans les feux de biomasse. Sa durée de vie étant de deux mois, le CO peut être transporté sur de longues distances. La mesure avec une bonne résolution spatiale et temporelle de $\mathrm{CO}$ est donc précieuse pour le suivi du temps chimique, c'est pourquoi le projet Macc $^{(1)}$ (pour le service atmosphérique de $\mathrm{GMES}^{(2)}$ ) en a fait l'un de ses produits essentiels.

Délivrée sur une base opérationnelle par Eumetsat, la colonne de monoxyde de carbone est un produit phare de Iasi. Les méthodes d'inversion pour CO ont été étudiées par de nombreuses équipes (Pommier, 2010 ; Illingsworth, 2010 ; Edwards, 2010). Le nombre de degrés de liberté est compris entre 0,8 et 1,8 . Dans les cas favorables, on peut donc retrouver deux sous-colonnes troposphériques, typiquement pour 0-5 km et $5-11 \mathrm{~km}$. En dehors d'Eumetsat qui utilise une méthode de réseaux neuronaux (August, 2010), la méthode d'inversion utilisée est une méthode d'estimation optimale. La différence de performance entre les différents algorithmes résulte principalement de l'information a priori utilisée, du filtrage des nuages et de la connaissance des profils atmosphériques et de la surface.

Des validations intensives ont été réalisées, principalement par comparaison avec le produit de l'instrument Mopitt (Measurements of Pollution in the Troposphere, instrument du satellite américain Terra), dédié à la cartographie de $\mathrm{CO}$, ou avec des sorties de modèle de chimie-transport. La précision pour la colonne totale est estimée à environ $10 \%$. La principale limitation vient de la méconnaissance des conditions de surface et des inversions de température.

(1) Macc: Monitoring Atmospheric Composition and Climate.

(2) GMES : Global Monitoring of Environment and Security.
Les cartes établies sur une base systématique par le LATMOS (Laboratoire, atmosphères, milieux, observations spatiales) sont transmises régulièrement au CEPMMT pour être utilisées dans Macc (figure 11).

Récemment, les cartes de CO établies avec Iasi ont permis d'illustrer l'extension des panaches et le transport du monoxyde de carbone lors des feux de Russie (George et al., 2010).

\section{Le dioxyde de carbone et le methane}

La restitution de la colonne de dioxyde de carbone $\left(\mathrm{CO}_{2}\right)$ avec une précision compatible avec les besoins de la communauté du cycle du carbone reste délicate, car les variations dues au $\mathrm{CO}_{2}$ sont de l'ordre de grandeur du bruit radiométrique. De plus, les radiances Iasi dans les bandes de $\mathrm{CO}_{2}$, comme dans tout l'infrarouge, dépendent en premier lieu du profil de température et toute incertitude sur cette variable vient rejaillir sur la concentration restituée. Néanmoins, l'utilisation simultanée des observations réalisées dans les micro-ondes par l'instrument Amsu compagnon de Iasi sur Metop-A, qui sont quant à elles sensibles à la température mais pas au $\mathrm{CO}_{2}$, permettent de restituer un contenu intégré de $\mathrm{CO}_{2}$ dans la haute troposphère (autour de 9-14 km) à l'aide d'une inférence non linéaire fondée sur des réseaux de neurones (Crevoisier et al., 2009a). Une moyenne sur des mailles de $5^{\circ}$ et sur un mois permet d'obtenir une précision d'environ 2 ppmv. L'analyse des cycles saisonniers et des tendances validées par des mesures aéroportées fournit d'importantes informations sur l'évolution du $\mathrm{CO}_{2}$ et son transport dans l'atmosphère (figure 12).

Concernant le méthane $\left(\mathrm{CH}_{4}\right)$, Iasi apporte, dans le meilleur des cas, 1,5 à 2 informations sur la verticale, localisées dans la troposphère. La colonne totale de méthane est restituée régulièrement par des méthodes de réseaux neuronaux (Crevoisier et al., 2009b) ou par régularisation. Les comparaisons avec des données du NDACC (Network for the Detection of Atmospheric Composition Change) montrent un biais de l'ordre de $1,5 \%$ et un écart quadratique moyen de 4,7 \% (Kerzenmacher et al., 2010).

\section{Les gaz traces mineurs}

La restitution d'espèces mineures constitue le résultat le plus novateur obtenu avec Iasi. Au total, le nombre d'espèces dont la signature a pu être mise en évidence avec Iasi est compris entre 20 et 25 (Coheur, 2010). Iasi est ainsi le premier instrument à proposer des mesures de l'ammoniac $\left(\mathrm{NH}_{3}\right)$ [Clarisse et al., 2009] permettant de détecter des régions cultivées contaminées par l'émission de ce gaz et de renseigner les modèles développés pour suivre l'évolution de cette pollution.

Iasi permet aussi la restitution de l'acide nitrique $\left(\mathrm{HNO}_{3}\right)$ présent en abondance dans l'UTLS.

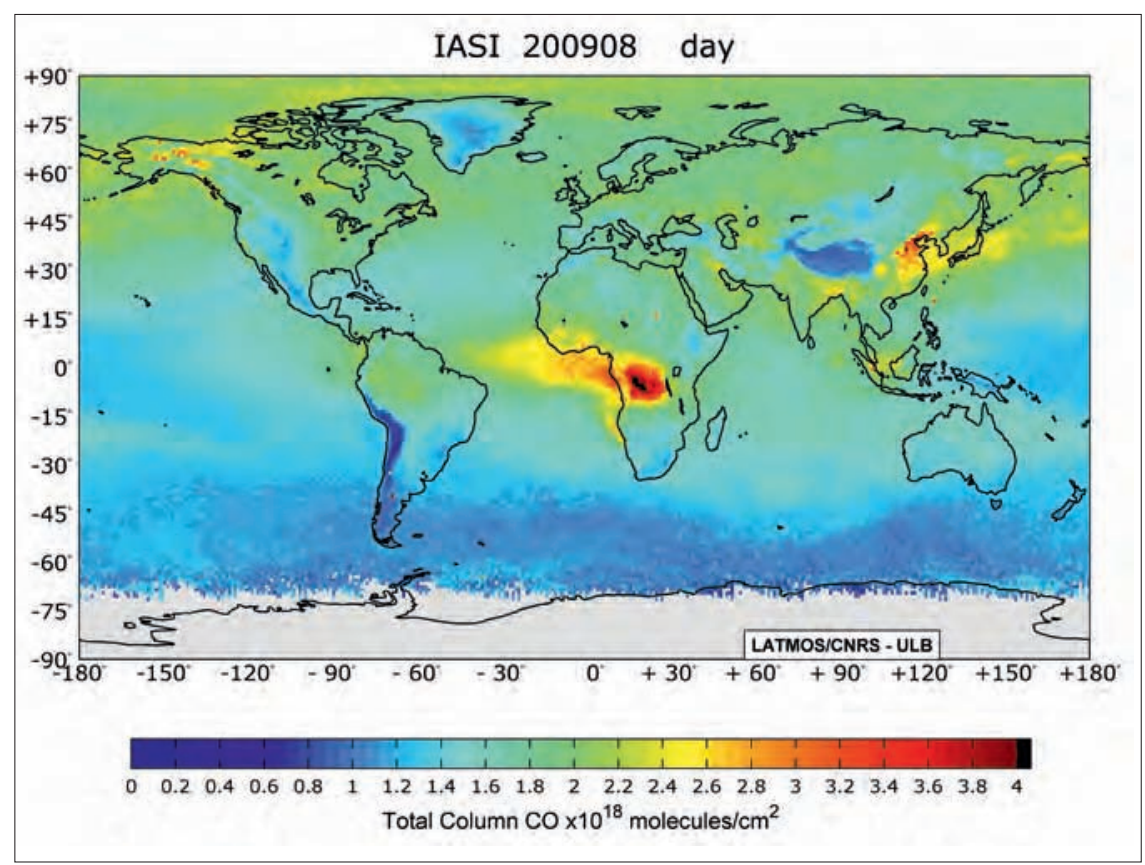

Figure 11 - Carte de mensuelle de CO (août 2009, mesures jour uniquement) illustrant le produit dérivé de lasi et transmis sur une base quotidienne à Macc. (৫ M. George) 


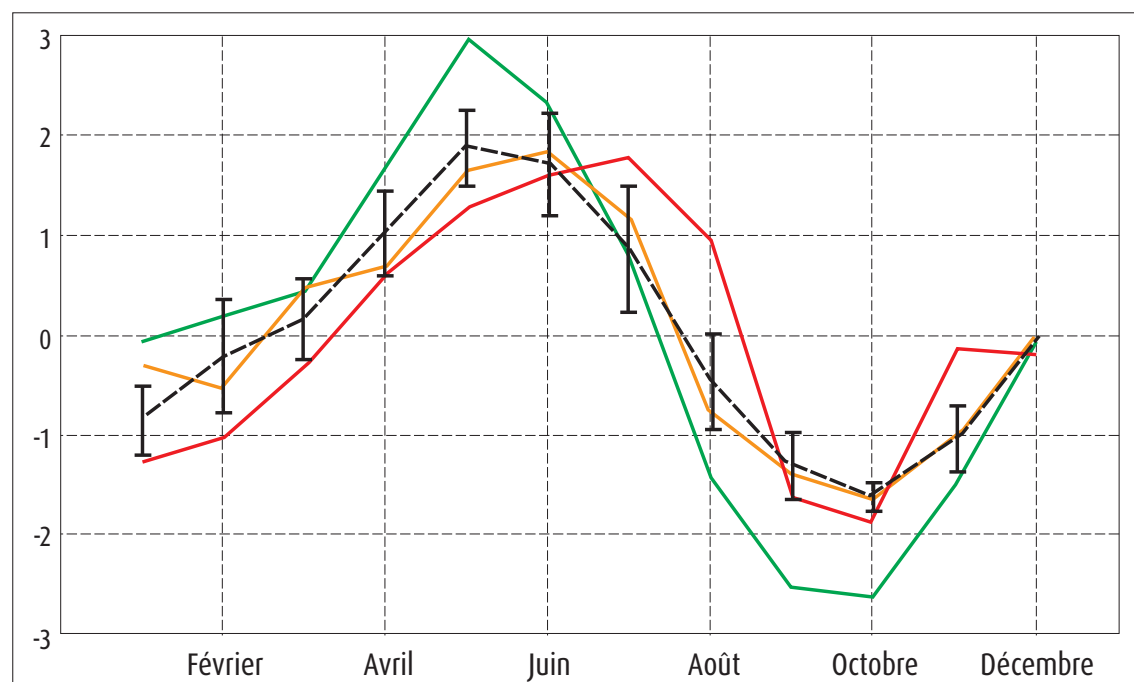

IASI 9-15 km (max ò $13 \mathrm{~km})$

JAM/CONTRAL aircraft 10km

AIRS 5-15 km (max ò $10 \mathrm{~km})$

MLO 4 km (GLOBALVIEW-2008)

Figure 12 - Cycle annuel du $\mathrm{CO}_{2}$ rapporté à la moyenne annuelle. Comparaison du $\mathrm{CO}_{2}$ lasi avec celui mesuré par I'Observatoire de Mauna Loa (MLO) à Hawaii, Airs et l'avion de la compagnie aérienne nationale japonaise JAL entre $0^{\circ}$ et $20^{\circ}$ de latitude Nord. Le décalage temporel est dû au transport vertical du $\mathrm{CO}_{2}$ (Crevoisier et al., 2010).

L'acide formique $(\mathrm{HCOOH})$ et le méthanol $\left(\mathrm{CH}_{3} \mathrm{OH}\right)$ dégagés par les feux de biomasse peuvent également être mis en évidence et quantifiés (Razavi et al., 2010 ; figure 13).

Pour le dioxyde de soufre $\left(\mathrm{SO}_{2}\right)$, Iasi tire parti des deux bandes d'absorption à 1150 et $1350 \mathrm{~cm}^{-1}$, pour détecter et quantifier les émissions de cette espèce lors des éruptions volcaniques et fournir une évaluation de l'altitude du panache (Karagulian et al., 2010 ; figure 14). Disposer de la continuité du spectre permet de distinguer le $\mathrm{SO}_{2}$ des aérosols de cendre et des nuages de glace, comme l'a prouvé le suivi des panaches volcaniques lors de l'épisode du volcan islandais Eyjafjallajökull.

\section{Les aérosols}

Certains aérosols comme les nuages de poussière désertique ( desert dust »), les nuages de cendres volcaniques, les nuages d'aérosols contenant de l'acide sulfurique/nitrique ou encore les panaches de feux peuvent être aisément détectés avec Iasi. En effet, les silicates qui entrent dans la composition des particules du type cendre ou poussière présentent des bandes d'absorption assez marquées dans l'infrarouge, notamment dans la région $1000-1300 \mathrm{~cm}^{-1}$. Les carbonates ont aussi cette particularité. La caractérisation de certains nuages comme ceux d'acide sulfurique ou des panaches de feux de biomasse est relativement aisée. Elle est fondée, en premier lieu, après détermination préalable de la structure de l'atmosphère en température et en vapeur d'eau, sur l'ajustement des deux caractéristiques essentielles que sont l'épaisseur optique et l'altitude moyenne de la couche de particules. On peut, dans une seconde étape, déterminer des paramètres microphysiques tels que la taille moyenne des particules. Cet ajustement peut se révéler difficile lorsqu'il y a des minimums locaux dans le processus de minimisation. De plus, la caractérisation des poussières désertiques reste plus complexe du fait d'atlas d'indices de réfraction incomplets ou de la granulométrie étendue en taille des particules qu'on trouve dans ces aérosols (Clarisse et al., 2010).

En revanche, un choix judicieux de canaux Iasi permet de restituer de façon très convenable l'épaisseur optique et l'altitude des aérosols absorbants. Une

Figure 13 - Variations saisonnières des distributions de l'acide formique, $\mathrm{HCOOH}$ (Razavi et al., 2011). Les valeurs les plus fortes observées dans les zones tropicales correspondent aux émissions de feux de biomasse.
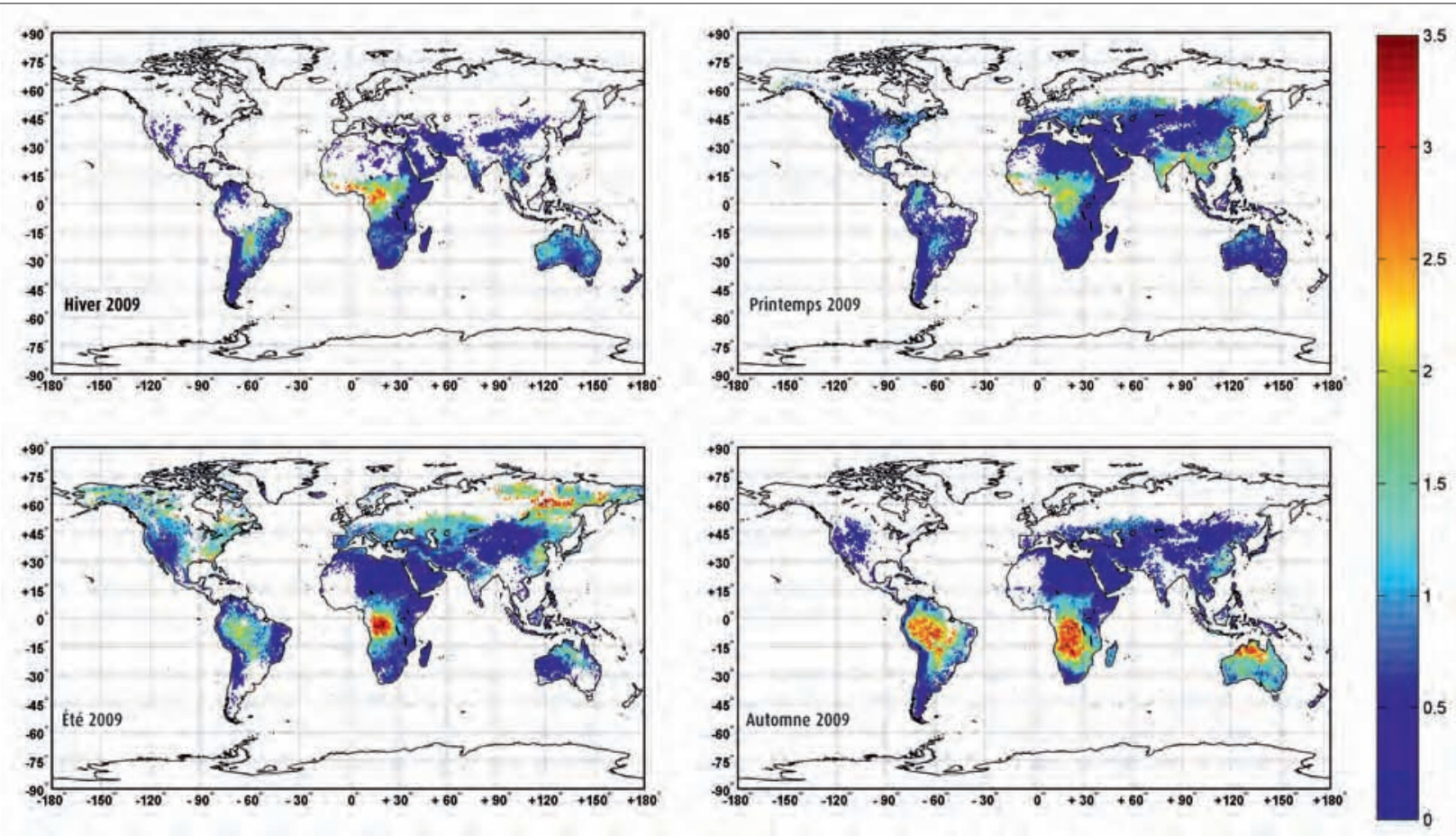


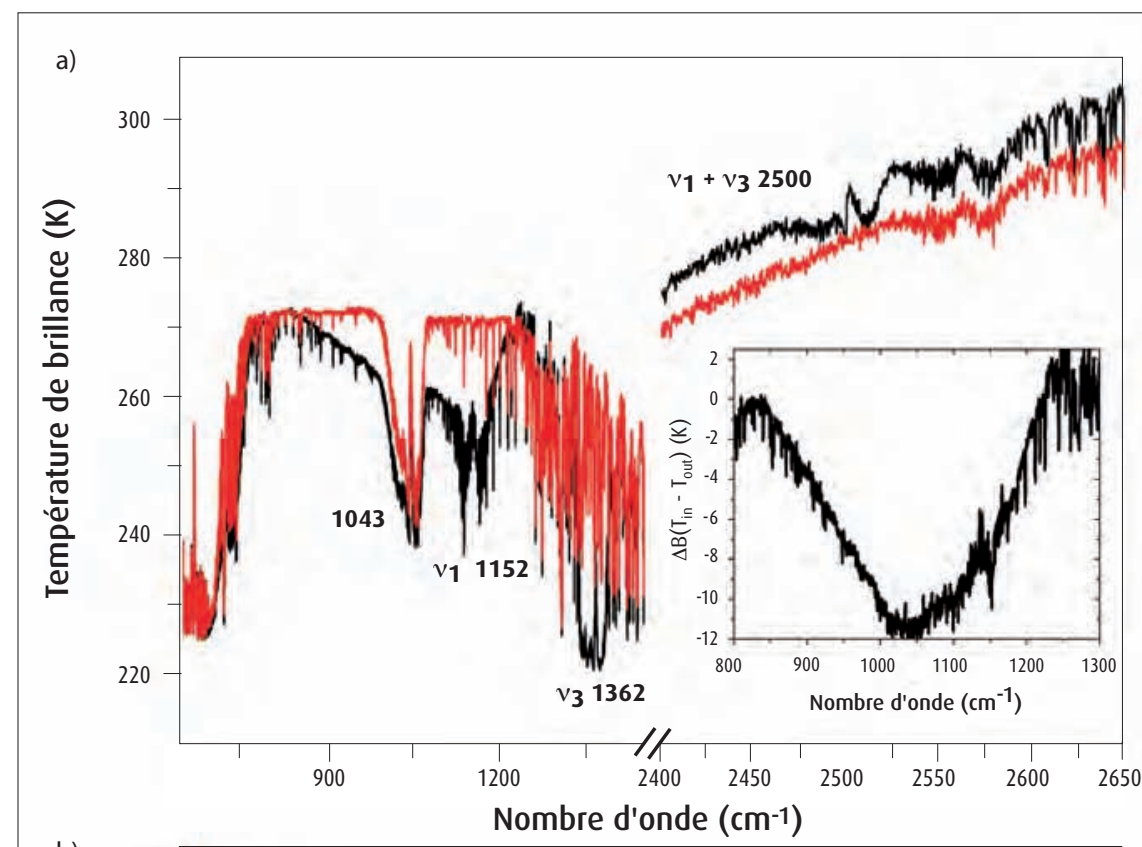

b)

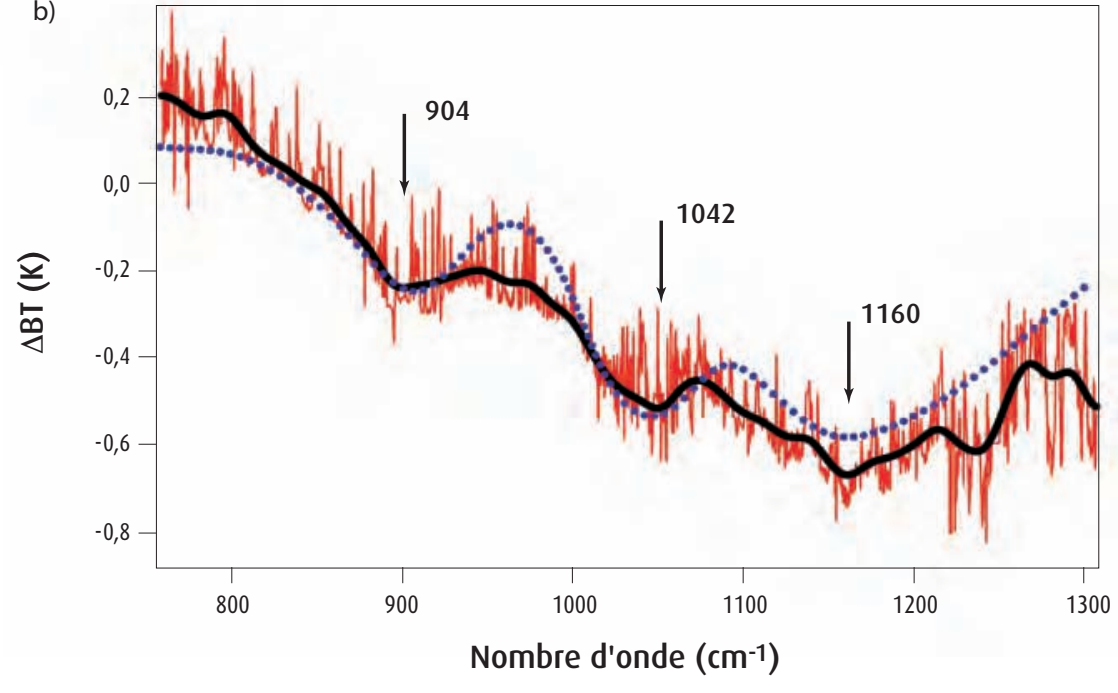

Figure 14 - Détection du $\mathrm{SO}_{2}$, des cendres et des aérosols de cendres et d'acide sulfurique lors de l'éruption volcanique du Kasatochi (îles Aléoutiennes en Alaska) :

a) les spectres lasi observés à côté (rouge) ou au-dessus du panache (noir) indiquent très clairement la présence d'aérosols de cendres en plus de gaz $\mathrm{SO}_{2}$ (absorption à $v_{1}=1152$ et $v_{3}=1362 \mathrm{~cm}^{-1}$ ) ;

b) un mois après, on peut détecter la présence d' $\mathrm{H}_{2} \mathrm{SO}_{4}$ (Karagulian et al., 2010). [C P.-F Coheur]

application intéressante et prometteuse est la climatologie des panaches de poussières désertiques (Peyridieu et al., 2010) dont on peut caractériser la nature ainsi que l'épaisseur optique, l'altitude et la distance à la zone source par transport atmosphérique.

\section{Apport pour des variables climatiques essentielles}

Le programme des satellites en orbite polaire (EPS) d'Eumetsat avec Metop s'étale sur 15 ans (avec trois modèles successifs de Iasi) et permet donc de constituer de longues séries temporelles, utilisations de ces profils atmosphériques, les études de variabilité du climat nécessitent de disposer de Thematic Climate Data Records (série temporelle de produits géophysiques homogénéisée d'un satellite à l'autre pour permettre le suivi de variables climatiques) issus d'un même système qui peuvent se montrer utiles, en complément des réanalyses. L'utilisation de Iasi pour faire des pseudo-radiosondages pour la prévision « experte » (travaux de Lavanant) reste une application très importante qui vise l'aide au prévisionniste. En outre, les profils sont utilisés en direct dans des modèles à mésoéchelle comme Arome. Les données de niveau 2 produites par Eumetsat sont également distribuées sur le réseau de diffusion des données météorologiques. Enfin, les engagements d'Eumetsat établis dans les spécifications de mission concernent ces produits et la recette impose une validation de ces profils par des moyens appropriés.

L'optimisation des techniques d'inversion reste une préoccupation de la communauté ainsi que la validation. Les méthodes utilisées peuvent être soit des méthodes statistiques fondées sur les composantes principales, soit une inversion non linéaire optimale - dite «1DVar »- (avec différentes méthodes de régularisation). Pour ces inversions, l'ébauche peut être fournie soit par une analyse du modèle soit par une prévision ou à partir d'une première inversion avec une méthode statistique (réseau de neurones ou régression sur des supercanaux). Un exercice d'intercomparaison des résultats d'inversion a été mené par l'ISSWG sur les données de validation de la campagne de mesures Jaivex (Hilton et al., 2009). La comparaison montre que les résultats sont plus ou moins semblables, et que la précision sur la température est tout à fait satisfaisante. En revanche, les variations verticales de l'humidité sont difficiles à restituer. Le choix de l'ébauche est essentiel pour ajuster au mieux le profil réel (Lavanant, 2010). Pougatchev (2009) a exploité les données de validation collectée à Lindenberg (Allemagne) pour affiner la validation des produits de niveau 2 d'Eumetsat (figure 15). Dans les basses couches, l'erreur de restitution reste assez forte pour l'humidité. Elle l'est aussi au-dessus des continents pour la température. Ce problème trouve ses causes dans la forte corrélation des radiances spectrales sensibles aux basses couches avec les radiances de surface. Il est dû aussi à l'incertitude sur l'émissivité et aux inversions de température fréquentes dans la couche limite atmosphérique. 

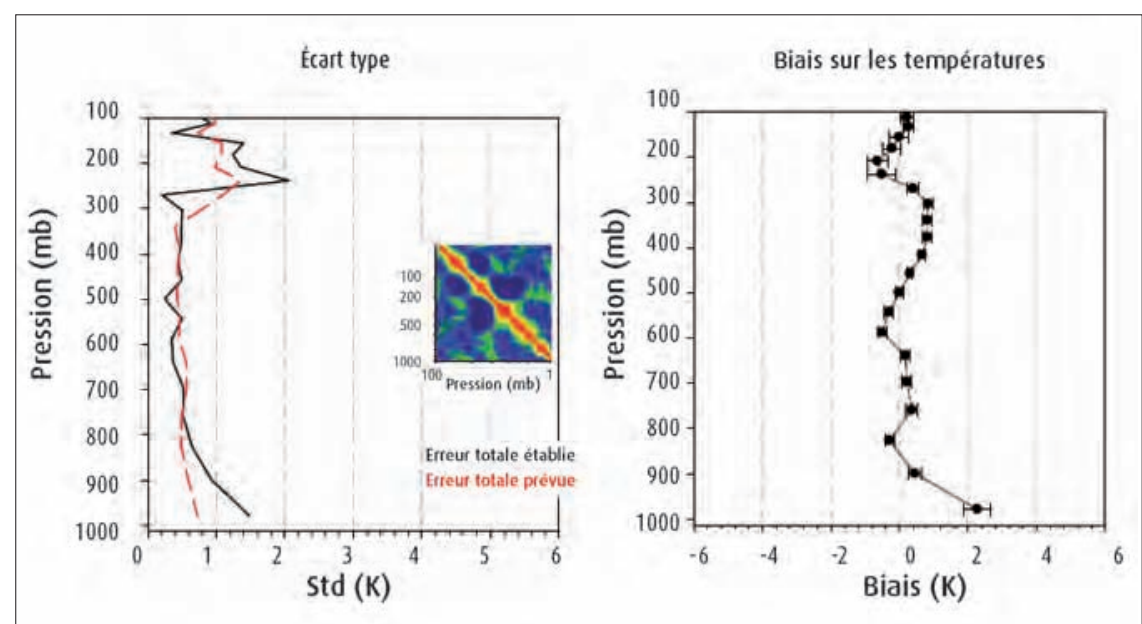

Figure 15 - Validation des profils de température déduits de lasi avec les sondages réalisés à Lindenberg en Allemagne (Pougatchev, 2010). À gauche l'écart type (en noir) et à droite l'erreur moyenne des écarts entre les profils restitués avec lasi par Eumetsat et les radiosondages réalisés à Lindenberg.

Pour ce qui concerne la température de surface, la précision sur mer est plutôt bonne. Sur les continents, elle est liée à la détermination de l'émissivité. Plusieurs équipes s'attachent à cette estimation. La technique repose sur une caractérisation des composantes principales liées à l'émissivité (Knuteson et Revercomb, 2004).

Pour les nuages, Eumetsat fournit comme produits la fraction nuageuse, la température de sommet du nuage, son niveau de pression, l'émissivité et la phase du nuage. Plusieurs équipes s'intéressent à la caractérisation des nuages soit à des fins climatologiques soit pour améliorer l'inversion en conditions nuageuses. Un exercice d'intercomparaison a été mis en place par l'ISSWG, coordonné par L. Lavanant. La synthèse fait ressortir des résultats assez similaires quant à la détection nuageuse. À ce stade, la référence choisie pour la couverture nuageuse reste celle donnée dans chaque pixel de l'imageur $\mathrm{AVHRR}^{(1)}$, présent avec Iasi sur le satellite Metop. Il y a plus de divergence dans l'analyse des situations où plusieurs couches nuageuses sont présentes. La comparaison des autres paramètres (pression au sommet des nuages) fait également apparaître des divergences. Il reste donc des progrès à faire pour la caractérisation des nuages. Des études sont menées pour accéder à des paramètre radiatifs ou microphysiques, avec des résultats encourageants.

\section{Le futur}

Deux autres modèles de vol Iasi seront lancés respectivement en 2012 et 2016. D'ici-là, les produits auront sans nul doute été améliorés et d'autres variables géophysiques auront été restituées. Néanmoins, les limites intrinsèques de Iasi pour ses diverses applications, qu'il s'agisse de résolution spatiale ou de performance radiométrique, sont d'ores et déjà perceptibles. Certains problèmes pourront être résolus grâce à l'information multitemporelle qui sera disponible avec le spectro-imageur infrarouge qui sera monté sur la $3^{\text {e }}$ génération de Meteosat. En dépit d'une résolution spectrale plus grossière que Iasi, ce capteur possède une meilleure résolution spatiale (4 km au sous-point satellite) et une répétitivité de $30 \mathrm{mn}$. Il permettra d'avoir une meilleure estimation des paramètres de surface et d'échantillonner de façon plus convenable les variations spatio-temporelles de l'humidité. Cependant ce capteur hyperspectral, avec une résolution spectrale de seulement $0,75 \mathrm{~cm}^{-1}$, ne permettra pas une description fine des profils verticaux.

En ce qui concerne les produits issus de Iasi, leur résolution verticale et leur précision ne pourront être améliorées que grâce à une meilleure résolution spectrale et à une meilleure performance radiométrique. La nouvelle génération de Iasi, Iasi-NG, spécifiée par la communauté scientifique et à l'étude au Cnes, offrira une résolution spectrale deux fois meilleure et un bruit radiométrique deux fois moindre que Iasi. Les premières simulations réalisées montrent les gains spectaculaires sur la précision des profils atmosphériques dans les basses couches et aussi les gains pour la chimie atmosphérique avec des profils plus précis et plus détaillés pour l'ozone et pour le $\mathrm{CO}$ ainsi que la quantification d'espèces pour l'instant seulement détectées avec Iasi (Clerbaux, 2010). Cette prochaine génération de sondeurs infrarou- ges est étudiée pour satisfaire les exigences de la communauté météorologique vis-à-vis des produits attendus de la génération de satellites polaires d'Eumetsat qui succédera à Metop pour la période 2020-2035.

\section{Conclusions}

Les résultats de Iasi, après trois ans d'exploitation, sont remarquables. Les tout premiers résultats mettaient en exergue l'impact positif des données Iasi sur la prévision. Les travaux pour améliorer cet impact se poursuivent dans des directions prometteuses avec l'utilisation de Iasi dans les régions nuageuses et au-dessus des terres émergées. Des résultats inattendus ont également été obtenus avec l'utilisation de Iasi dans des modèles à mésoéchelle, ce qui augure bien de l'impact attendu du sondeur infrarouge de Meteosat Third Generation à l'horizon 2017. La comparaison entre les profils atmosphériques restitués avec Iasi et des mesures in situ (dropsondes, radiosondages) montre que l'objectif de $1 \mathrm{~K} / 1 \mathrm{~km}$ est bien tenu sur mer mais qu'il subsiste des problèmes pour l'humidité (dans les basses couches). Les études pour améliorer ces produits se poursuivent.

Des résultats très spectaculaires ont été obtenus en chimie atmosphérique. La capacité de Iasi à restituer des profils d'ozone, avec des informations très utiles dans la basse troposphère ou aux alentours de la tropopause, a été démontrée. Les colonnes de CO issues de Iasi sont assimilées sur une base régulière dans GMES-Macc. En outre, Iasi est le premier instrument à détecter certaines espèces comme l'ammoniac et à permettre une analyse des panaches de feux et des espèces relâchées lors de ces événements. Les cartes de cendres volcaniques ont été largement montrées à l'occasion de l'éruption de l'Eyjafjallajökull. Enfin, pour les applications climatiques, la caractérisation des nuages et des surfaces (température, émissivité) est très satisfaisante mais nécessite d'être mieux valorisée.

Le potentiel de Iasi est certainement encore loin d'être totalement exploité. Les études se poursuivent pour améliorer les produits et étendre encore l'usage des données, d'autant que la

(1) AVHRR : radiomètre avancé à très haute résolution. C'est un instrument imageur multispectral. 
durée du programme Iasi (15 ans) laisse encore du temps pour exploiter l'instrument de façon optimale.

On commence cependant à mieux cerner les défis à relever pour la prochaine génération Iasi-NG dont le concept est à l'étude : exploitation en conditions nuageuses, amélioration des profils dans les basses couches, amélioration de la résolution verticale pour $\mathrm{O}_{3}, \mathrm{CO}, \mathrm{CH}_{4}$ ou $\mathrm{HNO}_{3}$ et quantification des espèces seulement détectées avec Iasi.

Pour finir, il importe de souligner l'avance considérable prise par l'Europe avec Iasi. Du côté américain, Airs est en fin de vie et Cris (Crosstrack Infrared Sounder), le sondeur hyperspectral qui ne volera sur le satellite NPP qu'à partir de 2011, est moins performant et ses capacités spectrales sont très réduites par rapport à Iasi. C'est pourquoi la NOAA (l'agence météorologique américaine), envisage très sérieusement d'équiper, à partir de 2025, ses satellites météorologiques polaires avec Iasi-NG.

\section{Bibliographie}

\footnotetext{
: Sur le site http://smsc.cnes.fr/IASI/A_conference.htm, sont disponibles les communications à la deuxième conférence internationale lasi qui s'est tenue du 24 au 29 janvier 2010 (à Sévrier en Haute-Savoie) des auteurs suivants : T. August, B. Barret, L. Buffet, L. Clarisse, C. Clerbaux, P. F. Coheur, C. Crevoisier, G. Dufour, - M. Eremenko, D. Edwards, L. Fiedler, N. Fourrié, M. George, V. Guidard, F. Hilton, T. Hultberg, S. Illingsworth, T. Kerzenmacher, J. Landgraf, L. Lavanant, A. McNally, S. Massart, S. Peyridieu, M. Pommier, J. Remedios, C. Scannel, L. Strow, D. Tobin et A. Wassmann.

Blumstein D., G. Chalon, T. Carlier, C. Buil, P. Hébert, T. Maciaszek, G. Ponce, T. Phulpin, B. Tournier, D. Siméoni, P. Astruc, A. Clauss, G. Kayal et R. Jegou, - 2004 : IASI instrument: technical overview and measured performances. Proc. SPIE, 5543, 196-207, DOI: 10.1117/12.560907.

- Blumstein D., B. Tournier, F.-R. Cayla, T. Phulpin, R. Fjortoft, C. Buil et G. Ponce, 2007 : In-flight performance of the Infrared Atmospheric Sounding Interferometer - (IASI) on Metop-A. Proc. SPIE, 6684, 66840H, DOI: 10.1117/12.734162.

- Boynard A., C. Clerbaux, P.-F. Coheur, D. Hurtmans, S. Turquety, M. George, J. Hadji-Lazaro, C. Keim et J. Meyer-Arnek, 2009 : Measurements of total and tropospheric ozone from IASI: comparison with correlative satellite, ground-based and ozone-sonde observations. Atmos. Chem. Phys., 9, 6255-6271, D0I: 10.5194/acp-9-6255-2009.

- Cayla F.-R., 2001 : L'interféromètre lasi, un nouveau sondeur satellitaire à haute résolution. La Météorologie, 8 série, 32, 23-39.

- Clarisse L., C. Clerbaux, F. Dentener, D. Hurtmans et P.-F. Coheur, 2009 : Global ammonia distribution derived from infrared satellite observations. Nature Geosci., 2, 479-483, D0I: 10.1038/ngeo551.

- Collard A. D., 2007 : Selection of IASI channels for use in numerical weather prediction. ECMWF Tech. Memorandum, 532.

- Crevoisier C., A. Chédin, H. Matsueda, T. Machida, R. Armante et N. A. Scott, 2009a : First year of upper tropospheric integrated content of CO ${ }_{2}$ from IASI hyper- spectral infrared observations. Atmos. Chem. Phys., 9, 4797-4810.

- Crevoisier C., D. Nobileau, A. M. Fiore, R. Armante, A. Chédin et N. A. Scott, 2009b : A new insight on tropospheric methane in the Tropics - first year from IASI - hyperspectral infrared observations. Atmos. Chem. Phys., 9, 6855-6887.

- George M., C. Clerbaux, D. Hurtmans, S. Turquety, P.-F. Coheur, M. Pommier, J. Hadji-Lazaro, D. P. Edwards, H. Worden, M. Luo, C. Rinsland et - W. McMillan, 2009 : Carbon monoxide distributions from the IASI/Metop mission: evaluation with other space-borne remote sensors. Atmos. Chem. Phys. , 9, 8317-8330, - DOI: 10.5194/acp-9-8317-2009.

: Goldberg M., G. Ohring, J. Butler, C. Cao, R. Datla, D. Doelling, V. Gärtner, T. Hewison, B. lacovazzi, D. Kim, T. Kurino, J. Lafeuille, P. Minnis, D. Renaut, - J. Schmetz, D. Tobin, L. Wang, F. Weng, X. Wu, F. Yu, P. Zhang et T. Zhu, 2011 : The Global Space-based Inter-Calibration System (GSICS). Bull. Amer. Met. Soc. - accepté pour publication.

- Han W. et A. P. McNally, 2010 : The 4D-Var assimilation of ozone-sensitive infrared radiances measured by lasi. Q. J. Roy. Meteorol. Soc. 136, 2025-2037.

- Hilton F., P. Antonelli, X. Calbert, T. Hultberg, L. Lavanant, X. Liu, D. Zhou, G. Masiello, C. Serio, S. Newman et J. Taylor, 2009 : An investigation into the per- formance of retrievals of temperature and humidity from IASI. Eumetsat P.55 - 2009 Eumetsat meteorological satellite Conference, Session 7.

: Karagulian F., L. Clarisse, C. Clerbaux, A. J. Prata, D. Hurtmans et P.-F. Coheur, 2010 : Detection of volcanic $\mathrm{SO}_{2}$, ash, and $\mathrm{H}_{2} \mathrm{SO}_{4}$ using the Infrared Atmospheric : Sounding Interferometer (IASI). J. Geophys. Res., 115, D00L02, D0I: 10.1029/2009JD012786.

: Knuteson R. et H. Revercomb, 2004 : Land surface temperature and emissivity from advanced sounders. Eumetsat P.41 - 2004 Eumetsat meteorological satellite - Conference, Session 2.

Pougatchev N., T. August, X. Calbet, T. Hultberg, O. Oduleye, P. Schlüssel, B. Stiller, K. S. Germain et G. Bingharn, 2009 : IASI temperature and water vapour - retrievals - error assessment and validation. Atmos. Chem. Phys. Discuss, 9, 7971-7989, D0I: 10.5194/acpd-9-7971-2009.

- Rabier F., A. Bouchard, E. Brun, A. Doerenbecher, S. Guedj, V. Guidard, F. Karbou, V.-H. Peuch, L. El Amraoui, D. Puech, C. Genthon, G. Picard, M. Town, A. Hertzog, F. Vial, P. Cocquerez, S. A. Cohn, T. Hock, J. Fox, H. Cole, D. Parsons, J. Powers, K. Romberg, J. VanHandel, T. Deshler, J. Mercer, J. S. Haase, L. Avallone, L. Kalnajs, C. R. Mechoso, A. Tangborn, A. Pellegrini, Y. Frenot, J.-N. Thépaut, A. McNally, G. Balsamo et P. Steinle, 2010 : The Concordiasi pro- ject in Antarctica. Bull. Amer. Meteor Soc., 91, 69-86. Traduction française : Le projet Concordiasi en Antarctique. La Météorologie, 8e série, 69, 42-55.

- Razavi A., F. Karagulian, L. Clarisse, D. Hurtmans, P.-F. Coheur, C. Clerbaux, J. F. Müller et T. Stavrakou, 2010 : First global distributions of methanol and formic - acid retrieved from the IASI/MetOp thermal infrared sounder. Atmos. Chem. Phys. Discuss., 10, 21475-21519.

- Schlüssel P., T. Hultberg, P. Phillips, T. August et X. Calbet, 2005 : The operational IASI Level 2 processor. Adv. Space Res., 36, 982-988.

- Siméoni D., P. Astruc, D. Miras, C. Alis, O. Andreis, D. Scheidel, C. Degrelle, P. Nicol B. Bailly, P. Guiard, A. Clauss, D. Blumstein, T. Maciaszek, G. Chalon, - T. Carlier et G. Kayal, 2004 : Design and development of IASI instrument. Proc. SPIE, 5543, 208-2109, D0I:10.1117/12.561090.
} 